\title{
Competition between Surface Layering and Surface Phase Formation in Dilute Liquid Hg-Au Alloys
}

\section{Citation}

DiMasi, E., H. Tostmann, B. M. Ocko, Peter S. Pershan, and M. Deutsch. 1999. Competition between surface layering and surface phase formation in dilute liquid $\mathrm{Hg}-\mathrm{Au}$ alloys. Journal of Physical Chemistry B 103(45): 9952-9959.

\section{Published Version}

doi:10.1021/jp9921553

\section{Permanent link}

http://nrs.harvard.edu/urn-3:HUL.InstRepos:4455228

\section{Terms of Use}

This article was downloaded from Harvard University's DASH repository, and is made available under the terms and conditions applicable to Other Posted Material, as set forth at http:// nrs.harvard.edu/urn-3:HUL.InstRepos:dash.current.terms-of-use\#LAA

\section{Share Your Story}

The Harvard community has made this article openly available.

Please share how this access benefits you. Submit a story.

\section{Accessibility}




\title{
Competition between Surface Layering and Surface Phase Formation in Dilute Liquid $\mathrm{Hg}-\mathrm{Au}$ Alloys
}

\author{
E. DiMasi, ${ }^{*}$, H. Tostmann,, B. M. Ocko, ${ }^{\dagger}$ P. S. Pershan, ${ }^{\ddagger}$ and M. Deutsch ${ }^{\S}$ \\ Department of Physics, Brookhaven National Laboratory, Upton, New York 11973-5000; Division of \\ Engineering and Applied Sciences and Department of Physics, Harvard University, \\ Cambridge, Massachusetts 02138; and Department of Physics, Bar-Ilan University, Ramat-Gan 52100, Israel
}

Received: June 25, 1999; In Final Form: August 17, 1999

\begin{abstract}
We present temperature-dependent X-ray reflectivity measurements of liquid $\mathrm{Hg}$ alloyed with $0.06-0.20$ atom $\% \mathrm{Au}$. At low Au concentrations, we find temperature-dependent surface-induced layering similar to that observed in pure $\mathrm{Hg}$, except that the presence of Au reduces the layering amplitude. Upon approaching the solubility limit of $\mathrm{Au}$ in $\mathrm{Hg}$, a new surface phase forms which is 1-2 atomic diameters thick and has a density of about half that of bulk $\mathrm{Hg}$. We present a surface phase diagram, summarizing the evolution of this unexpected surface structure upon varying composition and temperature. Such surface modifications may account for the variations observed in catalytic and electrochemical reactions at liquid metal surfaces upon alloying.
\end{abstract}

\section{Introduction}

Mercury and gold are among the few metals that can be found in their native state in nature and which were known to early civilizations. ${ }^{1}$ The use of $\mathrm{Hg}$ to extract $\mathrm{Au}$ and $\mathrm{Ag}$ from their ores, known as amalgamation, is one of the earliest metallurgical processes known to humanity. ${ }^{2}$ It is therefore no surprise that $\mathrm{Hg}-\mathrm{Au}$ amalgams have captured the interest of alchemists, metallurgists, chemists, physicists, and dentists. ${ }^{3} \mathrm{Hg}$ and $\mathrm{Au}$ are both transition metals with filled d-bands, are of similar atomic size, and form a variety of stable intermetallic phases in the solid state. ${ }^{4}$ The low cohesive energy of $\mathrm{Hg}$, evident from its low melting point of $-38.9^{\circ} \mathrm{C}$, enables these amalgams to form readily, simply by bringing $\mathrm{Au}$ into contact with liquid $\mathrm{Hg}$ under ambient conditions.

Despite a prevailing interest in $\mathrm{Hg}-\mathrm{Au}$ amalgams, not many detailed structural studies of $\mathrm{Hg}-\mathrm{Au}$ compounds have been performed. This may be attributed to the fact that it proves very difficult to establish the $\mathrm{Hg}-\mathrm{Au}$ phase diagram due to the high volatility of $\mathrm{Hg}$, which requires the use of sealed tubes at undetermined pressures for Hg-rich alloys. ${ }^{4}$ Much recent work has focused on surfaces of solid $\mathrm{Hg}-\mathrm{Au}$ amalgams. For example, microscopy and spectroscopy studies have addressed the morphology and composition of $\mathrm{Hg}-\mathrm{Au}$ phases formed by depositing $\mathrm{Hg}$ onto Au films. ${ }^{5-7}$ These studies are complicated by the morphology of the Au substrate and the coexistence of several $\mathrm{Hg}-\mathrm{Au}$ phases in the amalgam. ${ }^{7}$ Deposition of $\mathrm{Hg}$ onto $\mathrm{Au}$ electrodes has also been studied through electrochemical techniques. ${ }^{8}$ In-situ surface X-ray diffraction measurements of underpotential deposition of $\mathrm{Hg}$ onto the crystalline $\mathrm{Au}(111)$ electrode have revealed that amalgamation in the solid occurs in several steps, characterized by distinct surface phases. ${ }^{8}$ Since some of these phases are modified by coadsorbed anions from the electrolyte, and since kinetic effects produce further

* Author to whom correspondence should be addressed. E-mail: dimasi@ bnl.gov.

Brookhaven National University.

$\doteqdot$ Harvard University.

$\S$ Bar-Ilan University. complications, it is difficult to obtain basic structural information about the solid $\mathrm{Hg}-\mathrm{Au}$ amalgam through such studies.

In light of the knowledge about $\mathrm{Hg}-\mathrm{Au}$ phase formation, it is surprising that Au was once regarded as an "inert" material that could be used as part of a liquid $\mathrm{Hg}$ electrode in electrochemical studies. ${ }^{9}$ However, it was soon recognized that the potential of the amalgam electrode differed from that of pure $\mathrm{Hg}$. A more interesting observation was that small amounts of $\mathrm{Au}$ dissolved into the $\mathrm{Hg}$ droplet can strongly affect the electrochemical behavior by forming intermetallic compounds with other metals such as $\mathrm{Cd}$ and $\mathrm{Zn}$ that are present as impurities in the liquid $\mathrm{Hg} .{ }^{9}$ Metal impurities in liquid $\mathrm{Hg}$ have also been observed to affect the activation energies of reactions catalyzed by the liquid metal surface. ${ }^{10}$ It is not known whether such effects are due to changes in the electronic properties of liquid $\mathrm{Hg}$, modification of the surface structure, or the formation of intermetallic phases at the surface.

Until very recently, atomic-scale structural measurements on liquid metal surfaces have not been available, leaving such questions unresolved. Recent atomic-resolution surface scattering measurements have revealed the formation of a wide variety of structures in liquid metals and alloys. Elemental $\mathrm{Hg}$, Ga, and In exhibit surface-induced layering, in which atoms are stratified parallel to the liquid-vapor interface, ${ }^{11-15}$ a result long predicted by theory. ${ }^{16}$ This stratification of ions, with the corresponding oscillatory surface-normal density profile, is shown schematically in Figure 1. In liquid metal alloys, surface layering may compete with the formation of surface phases, making the structure more complex. Prominent examples are the monolayer surface segregation observed in $\mathrm{Ga}-\mathrm{In}^{17}$ and $\mathrm{Ga}-\mathrm{Sn},{ }^{18}$ and the temperature-dependent thick wetting layer that forms at the free surface of $\mathrm{Ga}-\mathrm{Bi}$ for temperatures above the monotectic point. ${ }^{19}$ In most of these cases, surface layering persists in the alloy, but is modified to varying extents by the surface phase. Competition between layering and surface phase formation is expected to be particularly important for systems such as $\mathrm{Hg}-$ $\mathrm{Au}$, where attractive heteroatomic interactions dictate the formation of intermetallic phases in the bulk solid. Such well- 


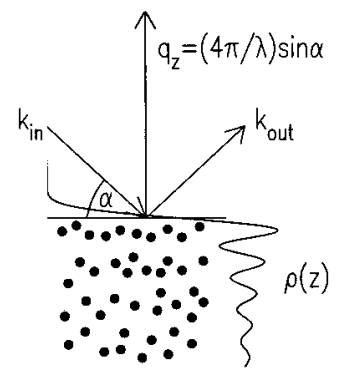

Figure 1. Geometry for X-ray reflectivity from a layered liquid metal surface. Maxima in the oscillatory surface-normal density profile $\rho$ $(z)$ correspond to layers of ions parallel to the liquid-vapor interface.

ordered phases in the solid typically give way upon melting to homogeneous mixtures in the bulk liquid. However, one may expect that the region close to the liquid-vapor interface, where order is induced in elemental liquid metals, may support the formation of intermetallic phases in the liquid alloy. Structural information for such systems is of fundamental interest and may well shed light on reactions occurring at liquid alloy surfaces, relevant to catalysis or electrochemistry.

In this paper we report X-ray reflectivity measurements of liquid $\mathrm{Hg}-\mathrm{Au}$ alloys having compositions near the room temperature saturation limit of 0.14 atom $\% \mathrm{Au}$ in $\mathrm{Hg},{ }^{4,20}$ at temperatures between +25 and $-39{ }^{\circ} \mathrm{C}$. We construct a temperature-concentration surface phase diagram and identify two distinct regions of surface phase behavior. At high $T$ and low Au concentrations, surface layering similar to that of pure $\mathrm{Hg}$ is observed. By contrast, at low $T$ and comparatively higher Au concentrations, we find evidence for the formation of a more complicated surface phase, where a new length scale for surface layering emerges along with a low-density layer at the interface.

\section{Experimental Details}

$\mathrm{X}$-ray reflectivity measurements were carried out using the Harvard-BNL liquid surface spectrometer at beamline X22B at the National Synchrotron Light Source, with an X-ray wavelength of $1.24 \AA$ and a detector resolution along the surface-normal direction of $0.035 \AA^{-1}$. The background intensity, due mainly to scattering from the bulk liquid, was subtracted from the specular signal by displacing the detector out of the reflection plane. The design of the liquid spectrometer $^{21}$ and a review of the measurement technique ${ }^{22}$ are given elsewhere.

Samples with nominal concentrations of 0.06, 0.10, 0.13, and 0.20 atom $\% \mathrm{Au}$ were produced by adding $\mathrm{Au}$ powder to liquid $\mathrm{Hg},{ }^{23}$ several days or weeks before the X-ray experiments. The samples were maintained at room temperature. The main experimental problem in surface measurements of metals is to ensure that a contaminant-free surface is obtained. The most reliable way to produce atomically clean metal surfaces is to keep them under ultra high vacuum (UHV) conditions, and to remove any residual oxide through argon ion sputtering. These UHV techniques, which we found to be very successful for $\mathrm{X}$-ray scattering measurements of $\mathrm{Ga}$, In, and low vapor pressure alloys, ${ }^{13,15,19}$ must be modified in the case of $\mathrm{Hg}$ and its alloys due to the high vapor pressure of $\mathrm{Hg}\left(\sim 10^{-2}\right.$ Torr at room temperature). For the measurements presented here, each sample was poured into a stainless steel reservoir within an argon-filled glovebox ( $<5$ ppm oxygen, $<2$ ppm water). The reservoir is connected to a stainless steel valve, filling capillary, and UHV flange and is affixed to a UHV compatible chamber. The empty chamber is evacuated to $10^{-7}$ Torr and baked out, after which the liquid $\mathrm{Hg}$ alloy is dropped from the reservoir through the
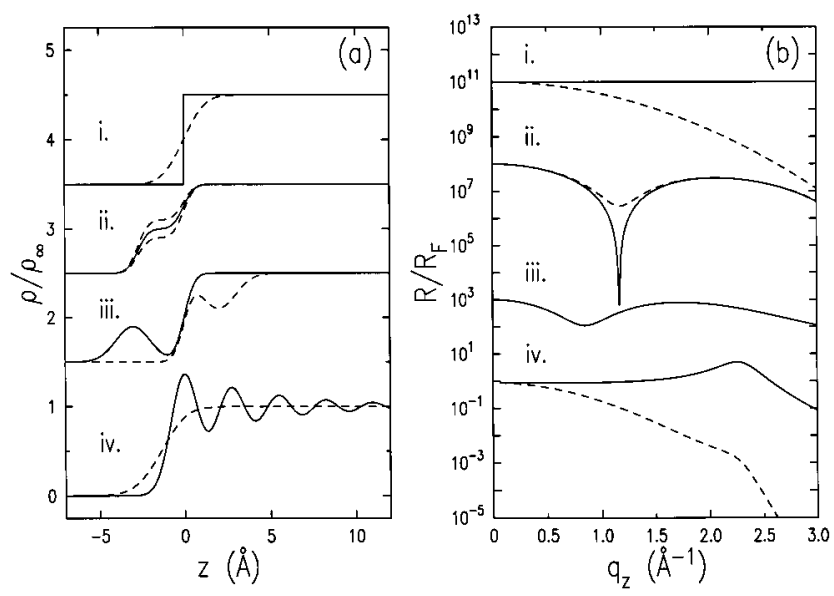

Figure 2. (a) Model density profiles and (b) corresponding Fresnelnormalized reflectivities as described in text. Curves $\mathrm{i}-$ iii in both panels are shifted for clarity.

valve and capillary into a ceramic sample pan within the chamber, as was done previously for measurements of pure Hg. ${ }^{12}$ Immediately after opening the valve to the reservoir, the $\mathrm{Hg}$ vapor pressure determines the total pressure in the chamber, while the partial pressures of possible contaminants such as water and oxygen remain low. The resulting liquid alloy surfaces were found to be stable for over a week, due to two mechanisms. First, $\mathrm{Hg}$ oxide introduced from pouring the sample is unstable and decomposes under these low oxygen partial pressures. ${ }^{24,25}$ In addition, slight but continual evaporation of $\mathrm{Hg}$ constantly refreshes the surface. ${ }^{26}$ Preliminary reflectivity measurements utilizing a glass sample chamber evacuated to $10^{-4}$ Torr and backfilled with dry hydrogen gas were qualitatively similar, but not sufficiently reproducible.

The samples were cooled with a liquid nitrogen cold finger beneath the sample pan. Temperatures were monitored at the sample pan and calibrated to that of the liquid surface in separate experiments using thermocouples immersed in the samples. The calibration has an uncertainty of $\pm 1{ }^{\circ} \mathrm{C}$.

\section{X-ray Reflectivity and Modeling}

$\mathrm{X}$-ray reflectivity is a powerful technique for investigating structure normal to surfaces and interfaces on atomic length scales. ${ }^{22,27-29}$ In these measurements, the scattered intensity is measured as a function of momentum transfer $q_{z}=k_{\text {out }}-k_{\text {in }}$ perpendicular to the surface (Figure 1) and normalized to the incident photon intensity.

The simplest interface is a step function describing a sharp truncation of a homogeneous bulk density $\rho_{\infty}$ (Figure $2 \mathrm{a}(\mathrm{i})$, solid line). Scattering from this sharp interface takes the form of the Fresnel reflectivity

$$
R\left(q_{z}\right)=\left[\frac{q_{z}}{q_{\mathrm{c}}}+\sqrt{\left(\frac{q_{z}}{q_{\mathrm{c}}}\right)^{2}-1}\right]^{-4} \equiv R_{\mathrm{F}}
$$

In this expression $R_{\mathrm{F}}$ depends on the bulk density $\rho_{\infty}$ through the critical wave vector $q_{\mathrm{c}}=\left(16 \pi \rho_{\infty} r_{0}\right)^{1 / 2}$, where $r_{0}=e^{2} / m c^{2}$ $=2.82 \times 10^{-5} \AA$ is the classical electron radius, and absorption has been neglected. For a surface-normal density profile that deviates from the step function, the reflectivity is modified from the Fresnel form. When the scattered intensity is much less than the incident intensity (generally the case as long as $q_{\mathrm{z}} \gtrsim 5 q_{\mathrm{c}}$ ), the kinematic approximation is valid and the reflectivity may be written 


$$
R\left(q_{z}\right)=R_{\mathrm{F}}\left|\frac{1}{\rho_{\infty}} \int_{-\infty}^{\infty}(\partial \rho / \partial z) \exp \left(i q_{z} z\right) \mathrm{d} z\right|^{2}
$$

which is sensitive to $\rho(z)$, the surface-normal density profile averaged laterally over the resolution defined X-ray correlation length. ${ }^{21}$ Since the phase information is not accessible to the experimental intensity measurement, eq 2 cannot be inverted to determine the density profile directly. For this reason the usual practice is to construct a model density profile and compare its calculated reflectivity to the experiment. Because of the phase problem, distinct real-space structural models can produce essentially identical reflectivity curves, lending some ambiguity to the analysis. ${ }^{30}$ Nevertheless, the ambiguities can often be resolved by consideration of basic physical constraints.

The simplest physically reasonable modification of the step function profile takes surface roughness into account. In a liquid, thermally excited capillary waves produce height variations across the surface, which are averaged in the scattering measurement over length scales determined by the resolution of the spectrometer. These occur in addition to any roughness intrinsic to the local surface-normal profile. Detailed discussions of the effect of capillary waves on the scattering cross section are given elsewhere. ${ }^{15,21}$ The capillary wave roughness $\sigma_{\mathrm{cw}}$ is a function of the temperature $T$ and the surface tension $\gamma$ and is given by

$$
\sigma_{\mathrm{cw}}^{2}=\frac{k_{\mathrm{B}} T}{2 \pi \gamma} \ln \left(\frac{q_{\mathrm{max}}}{q_{\mathrm{res}}}\right)
$$

This expression ${ }^{31}$ has been arrived at by integrating over those capillary modes having wavevectors less than $q_{\max }=\pi / a$, where $a$ is the atomic diameter, and greater than $q_{\text {res }}$, determined by the instrumental resolution.

We will consider a more general broadening of the density profile, by $\sigma_{\mathrm{T}}^{2}=\sigma_{\mathrm{cw}}{ }^{2}+\sigma_{\mathrm{i}}^{2}$, to incorporate the capillary wave roughness $\sigma_{\mathrm{cw}}$ along with any other roughness $\sigma_{\mathrm{i}}$ intrinsic to the surface. This profile, having

$$
\partial \rho / \partial z=\exp \left(-\sigma_{\mathrm{T}}{ }^{2} z^{2} / 2\right)
$$

is shown by dashed lines in Figure $2 \mathrm{a}(\mathrm{i})$, for $\sigma_{\mathrm{T}}=1.0 \AA$. The density profile can be written in terms of the error function:

$$
\rho(z)=\left(\rho_{\infty} / 2\right)\left[\operatorname{erf}\left(z / \sigma_{\mathrm{T}} \sqrt{2}\right)+1\right] \equiv \rho_{\text {erf }}
$$

yielding for the reflectivity

$$
R=R_{\mathrm{F}} \exp \left(-q_{z}^{2} \sigma_{\mathrm{T}}^{2}\right)
$$

The resulting Fresnel-normalized reflectivity is shown in Figure $2 \mathrm{~b}(\mathrm{i})$, dashed line. The exponential factor in eq 5 is analogous to the Debye-Waller term used to describe the reduction of the diffracted intensity from crystals due to thermal displacements of the atoms from the lattice sites. ${ }^{32}$

When more complicated surface-normal density profiles must be constructed, two straightforward methods are commonly used. Regions of differing electron density can be represented by a combination of error functions, parametrizing the thickness, density, and roughness between each region. A general profile of this type may be written

$$
\rho(z)=\sum_{n=0}^{N}\left(\frac{\rho_{n}-\rho_{n+1}}{2}\right)\left[\operatorname{erf}\left(\frac{z-z_{n}}{\sigma_{n} \sqrt{2}}\right)\right]
$$

with $\rho_{0}$ equal to the bulk density $\rho_{\infty}, \rho_{N+1}=0$ representing the vapor, and $\sigma_{n}$ describing the broadening between regions $n$ and $n+1$ at position $z_{n}$. The reflectivity for this model is

$$
R / R_{\mathrm{F}}=\left|\sum_{n=0}^{N}\left(\frac{\rho_{n}-\rho_{n+1}}{\rho_{\infty}}\right)\left[\exp \left(-q_{z}^{2} \sigma_{n}^{2} / 2+i q_{z} z_{n}\right)\right]\right|^{2}
$$

Figure $2 \mathrm{a}$ (ii) shows three such profiles, having one additional slab of low density between the vapor and the bulk liquid. Here $\sigma_{0}=\sigma_{1}=0.5 \AA$ and $\left|z_{1}\right|=2.7 \AA$. With the same roughness for both interfaces, the reflectivity at $q_{z}=\pi /\left|z_{1}\right|$ has an amplitude proportional to $\left(1-2 \rho_{1} / \rho_{0}\right)^{2}$. When $\rho_{1} / \rho_{0}=1 / 2$ (Figure $2 \mathrm{a}$ (ii), solid line), the result is total destructive interference at $q_{z}=$ $\pi /\left|z_{1}\right|$, producing a deep notch in the reflectivity (Figure $2 \mathrm{~b}$ (ii), solid line). For values of $\rho_{1}$ symmetrical about $1 / 2$, identical reflectivity curves are obtained. Dashed lines in Figure 2a(ii) show profiles having $\rho_{1} / \rho_{0}=0.4$ and 0.6 , which both produce the reflectivity curve shown as a dashed line in Figure $2 b$ (ii). This is an example of the unavoidable ambiguity in interpreting reflectivity measurements even for simple models.

An alternative method of constructing a structured surface profile is to define the density through a combination of Gaussian and error functions, as shown in Figure 2a(iii). For the example shown

$$
\rho(z)=\rho_{\text {erf }}+\frac{h_{\mathrm{g}} / \sigma_{\mathrm{g}}}{\sqrt{2 \pi}} \exp \left[-\left(z-z_{\mathrm{g}}\right)^{2} / 2 \sigma_{\mathrm{g}}^{2}\right]
$$

and

$$
\begin{aligned}
R / R_{\mathrm{F}}=\mid & \left.\mid \exp \left(-q_{z}^{2} \sigma_{\mathrm{T}}^{2} / 2\right)\right]^{2}+ \\
& \left.\frac{h_{\mathrm{g}}}{\rho_{\infty}} q \exp \left(-q_{z}^{2} \sigma_{\mathrm{g}}^{2} / 2\right) \exp \left[i\left(q_{z} z_{\mathrm{g}}-\pi / 2\right)\right]\right|^{2}
\end{aligned}
$$

The Gaussian position $z_{\mathrm{g}}$ and the sign of its amplitude $h_{\mathrm{g}}$ enter only in the cross term of eq 9, which is proportional to $h_{\mathrm{g}} \sin \left(q_{z} z_{\mathrm{g}}\right)$. For this reason, the reflectivity measurement does not distinguish between a positive Gaussian density added to the vapor side at position $z=z_{\mathrm{g}}$ (Figure 2a(iii), solid line) and density subtracted from the liquid side at $z=-z_{\mathrm{g}}$ (Figure $2 \mathrm{a}-$ (iii), dashed line). These two rather different looking density profiles both produce the reflectivity curve shown in Figure 2b(iii). The effect of this Gaussian term can be similar to that of a low-density surface slab: both produce minima in the reflectivity at low $q_{z}$, as shown by comparison of Figure $2 \mathrm{~b}$ (iii) and Figure 2b(ii), dashed line.

The models discussed so far describe a liquid-vapor interface having structure near the surface. To describe the surfaceinduced layering of liquid metals, which extends further into the bulk, a damped oscillatory surface-normal density profile must be constructed. A convenient way to do this is to model layers of atoms parallel to the surface by a series of Gaussian terms, representing mean-squared displacements of atoms assigned to each layer at spacing $d$ :

$$
\rho(z)=\rho_{\infty} \sum_{n=0}^{\infty} \frac{d / \sigma_{n}}{\sqrt{2 \pi}} \exp \left[-(z-n d)^{2} / 2 \sigma_{n}^{2}\right]
$$

With the choice of $\sigma_{n}^{2}=\sigma_{T}^{2}+n \bar{\sigma}^{2}$, we parametrize two effects in a simple way. First, the surface layers become less welldefined with increasing depth into the bulk liquid, at a rate controlled by $\bar{\sigma}$. Second, the effect of increasing $\sigma_{\mathrm{T}}$ is to reduce the overall layering amplitude, an effect expected both from 
capillary waves and from any roughness intrinsic to the local surface-normal profile.

The corresponding reflectivity is given by

$$
\begin{array}{r}
R / R_{\mathrm{F}}=\left[F_{Z}\left(q_{z}\right)\right]^{2}\left(q_{z} d\right)^{2}\left(-{\sigma_{T}}^{2} q_{z}^{2}\right)\left[1-2 \exp \left(-q_{z}^{2} \bar{\sigma}^{2} / 2\right)\right. \\
\left.\cos \left(q_{z} d\right)+\exp \left(-q_{z}^{2} \bar{\sigma}^{2}\right)\right]^{-1}
\end{array}
$$

Since each Gaussian now explicitly represents a collection of scattering atoms, here it is meaningful to incorporate the reduced atomic form factor $F_{Z}\left(q_{z}\right)=\left[f_{Z}\left(q_{z}\right)+f_{Z}^{\prime}\right] /\left[Z+f_{Z}^{\prime}\right]$ for atoms having atomic number $Z$. Figure $2 \mathrm{a}(\mathrm{iv})$ (solid line) illustrates a layered profile with $d=2.7 \AA, \sigma_{\mathrm{T}}=0.8 \AA$, and $\bar{\sigma}=0.45 \AA$. The reflectivity (Figure $2 \mathrm{~b}(\mathrm{iv})$, solid line) shows the broad quasi-Bragg peak produced by the oscillatory density profile. In the limit where $\sigma_{\mathrm{T}}$ is large, the profile approaches the error function form of eq 4, though with its argument shifted from $z$ $=0$ to $z=-d / 2$ due to the choice of origin in eq 10. A layered profile having $\sigma_{\mathrm{T}}=1.5 \AA$ is shown by dashed lines in Figure $2 \mathrm{a}(\mathrm{iv})$. Although the intensity falls off quickly for large $q_{z}$ (Figure 2b(iv), dashed line), constructive interference in the region of the layering peak is still evident.

Finally, this profile can be modified by additional Gaussian terms, and by changing the amplitudes, positions, and widths of the terms in eq 10. The most general model that we will present in this study takes the form

$$
\begin{aligned}
\rho(z) / \rho_{\infty}=\left(\frac{w_{\mathrm{A}} d}{\sigma_{\mathrm{A}} \sqrt{2 \pi}}\right) & \exp \left[-\left(z-z_{\mathrm{A}}\right)^{2} / \sigma_{\mathrm{A}}{ }^{2}\right]+ \\
& \sum_{n=0}^{\infty}\left(\frac{w_{n} d}{\sigma_{n} \sqrt{2 \pi}}\right) \exp \left[-(z-n d)^{2} / \sigma_{n}^{2}\right]
\end{aligned}
$$

where the Gaussian scaled by $w_{\mathrm{A}}$ is typically much broader than those controlled by $\sigma_{n}$ in the sum, and $w_{n}$ may deviate from unity. Since the effect of the extra Gaussian is similar to that of the Gaussian plus error function model described by eq 8 , placing this term into the vapor side of the interface has an effect which can be difficult to distinguish from that of decreasing the weights $w_{n}$ of the first few terms in the sum.

\section{Experimental Results}

Distinctly different reflectivities are obtained depending on whether the Au concentration exceeds the solubility limit. The solubility of $\mathrm{Au}$ in $\mathrm{Hg}$ as determined from macroscopic measurements ${ }^{20,33}$ is plotted as a function of temperature in Figure 3 (open circles; the shaded band is a guide for the eye). Measured normalized reflectivity curves are shown for a selection of temperature and concentration points, identified by small crosses on the graph. All $R / R_{\mathrm{F}}$ curves are shown on a semilog scale with ranges identical to those shown for 0.13 atom $\%$ Au at $-18{ }^{\circ} \mathrm{C}$. This phase diagram provides an overview of our results, which will be discussed in more detail further below.

At low Au concentrations and high temperatures (upper right region of Figure 3), the reflectivity is characterized mainly by the layering peak at $q_{z} \approx 2.2 \AA^{-1}$. At the lowest Au concentration, 0.06 atom $\% \mathrm{Au}$, the reflectivity is very similar to that of pure $\mathrm{Hg}$. The data for alloys below the solubility limit are shown in Figure 4. Solid lines are fit curves that will be described in the Analysis and Discussion section. For both $\mathrm{Hg}$ and $\mathrm{Hg} 0.06$ atom $\% \mathrm{Au}$, the effect of increased temperature is to reduce the amplitude of the interference peak, from the lowest temperature measured, $-26{ }^{\circ} \mathrm{C}$, up to $+25{ }^{\circ} \mathrm{C}$. Repeated cooling and

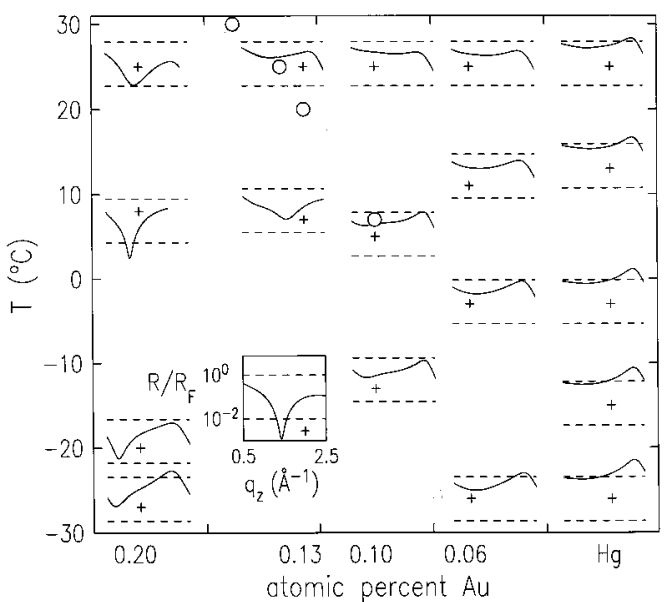

Figure 3. Surface phase diagram for liquid $\mathrm{Hg}-\mathrm{Au}$ alloys, with nominal $\mathrm{Au}$ concentrations from $0-0.20$ atom $\%$ and temperatures between -28 and $+25{ }^{\circ} \mathrm{C}$, indicated by crosses $(+) . R / R_{\mathrm{F}}$ curves are shown on semilog scales, all with identical axes as marked for the 0.13 atom $\%,-18{ }^{\circ} \mathrm{C}$ alloy. (O) Reported solubility limit of $\mathrm{Au}$ in $\mathrm{Hg}$ (refs 20 and 33). The shaded band is a guide for the eye.

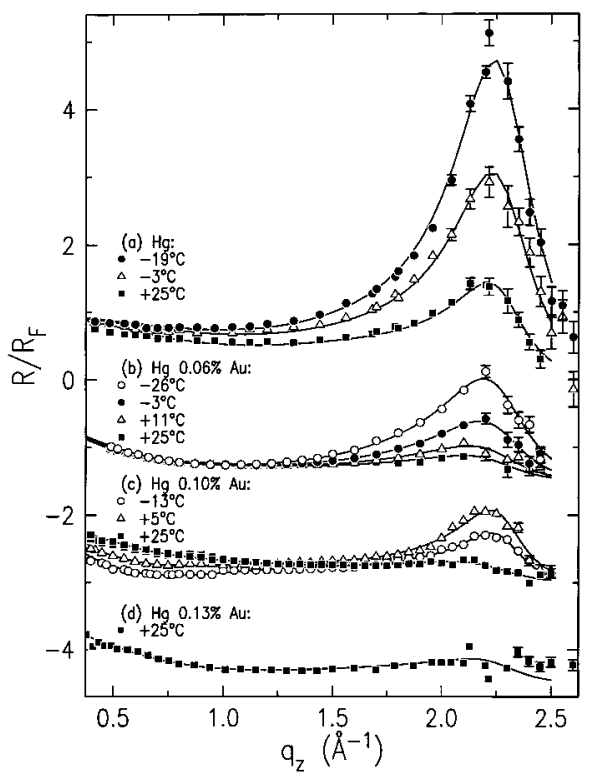

Figure 4. Fresnel-normalized reflectivity of dilute $\mathrm{Hg}-\mathrm{Au}$ alloys for selected temperatures: (a) pure $\mathrm{Hg}$, from ref 12; (b) $\mathrm{Hg} 0.06$ atom \% $\mathrm{Au}$; (c) $\mathrm{Hg} 0.10$ atom \% Au; (d) $\mathrm{Hg} 0.13$ atom \% Au. Curves b-d are shifted for clarity. Solid lines are fits as discussed in text.

warming between these temperatures showed that the effects of $T$ are completely reversible for $\mathrm{Hg}$ and $\mathrm{Hg} 0.06$ atom \% Au. The data are consistent with scattering from a layered liquid metal surface, where the effects of the atomic layering are partially disrupted by thermal excitations. For 0.10 atom $\% \mathrm{Au}$ in $\mathrm{Hg}$, comparable behavior is observed at +25 and $+5^{\circ} \mathrm{C}$. On cooling further to $-13{ }^{\circ} \mathrm{C}$, however, the amplitude of the layering peak decreases, showing that surface layering is being suppressed at lower temperatures. The reflectivity in the region $q_{z} \sim 0.3-1.0 \AA^{-1}$ also changes with $T$, unlike the case of the more dilute alloy. This means that the structure within $2 \AA$ or so of the interface changes with $T$ at the higher concentration of 0.10 atom $\% \mathrm{Au}$. This is not the case for pure $\mathrm{Hg}$ and for $\mathrm{Hg} 0.06$ atom \% Au, where the near-surface structure is temperature independent. The behavior of the 0.10 atom $\%$ alloy in this temperature range marks the transition between viability of the surface layering, which is enhanced at lower temperatures, and the formation of a competing surface structure. Similarly, 


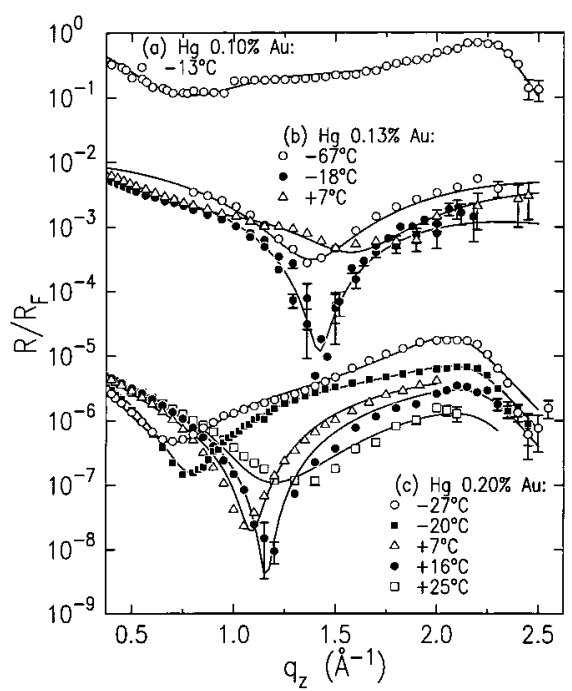

Figure 5. Fresnel-normalized reflectivity of $\mathrm{Hg}$-rich alloys: (a) $\mathrm{Hg}$ 0.10 atom $\% \mathrm{Au}$ at $-13{ }^{\circ} \mathrm{C}$; (b) $\mathrm{Hg} 0.13$ atom \% Au below room temperature; (c) $\mathrm{Hg} 0.20$ atom \% Au, first cool-down from room temperature. Curves b and $\mathrm{c}$ are shifted by factors of $10^{-2}$ and $10^{-5}$, respectively. Solid lines are fits as discussed in text.

the reflectivity from $\mathrm{Hg} 0.13$ atom \% Au can be described by a simple surface layering model only at room temperature, and is substantially different at lower temperatures.

The data are qualitatively different when the Au concentration exceeds the solubility limit in liquid $\mathrm{Hg}$ (bottom left region in Figure 3). For 0.13 atom $\% \mathrm{Au}$ in $\mathrm{Hg}$, below room temperature, the reflectivity exhibits a deep minimum, which shifts with decreasing temperature, from $q_{z} \approx 1.6$ at $+7{ }^{\circ} \mathrm{C}$ to $q_{z} \approx 1.4$ $\AA^{-1}$ at $-67{ }^{\circ} \mathrm{C}$ (Figure 5b). The position and depth of this minimum suggest the formation of a surface region having about half the density of the bulk, which is about one atomic diameter thick and which grows slightly thicker at lower temperatures. This trend with temperature is confirmed more dramatically in reflectivity measurements of 0.20 atom $\% \mathrm{Au}$ in $\mathrm{Hg}$, where the Au concentration exceeds the solubility limit at all temperatures. The notch in the reflectivity is present at room temperature, and shifts in $q_{z}$ from $1.3 \AA^{-1}$ at room temperature to $0.6 \AA^{-1}$ at $-27{ }^{\circ} \mathrm{C}$ (Figure 5c). Even more interesting, at the lowest temperatures a peak has appeared which resembles the layering peak of $\mathrm{Hg}$, but at measurably smaller $q_{z}$ than for $\mathrm{Hg}$. At these two highest $\mathrm{Au}$ concentrations, temperature cycling results in hysteresis and long equilibration times. After cooling the 0.13 atom $\%$ alloy close to its melting point and heating to room temperature again, approximately $10 \mathrm{~h}$ at room temperature were required to reproduce the reflectivity. With 0.20 atom $\% \mathrm{Au}$, the original room temperature reflectivity was never recovered after cooldown, even after $50 \mathrm{~h}$ at $+25{ }^{\circ} \mathrm{C}$. Reflectivity data for the second cool-down of this alloy are shown in Figure 6. The reflectivity curves for the second cool-down all exhibit the notch at lower $q_{z}$ for each temperature than the first measurements, until the melting point is approached. Au expelled from the bulk, presumably incorporated into the surface structure, apparently cannot readily resolubilize at these concentrations.

\section{Analysis and Discussion}

A. Fit Models. By fitting reflectivity curves calculated from model surface-normal density profiles to the data, we can obtain more specific information about the surface structure of these alloys. Nearly all of the experimental reflectivity curves exhibit an interference peak along with some additional structure at lower $q_{z}$. We have fit these measurements to a simple layered

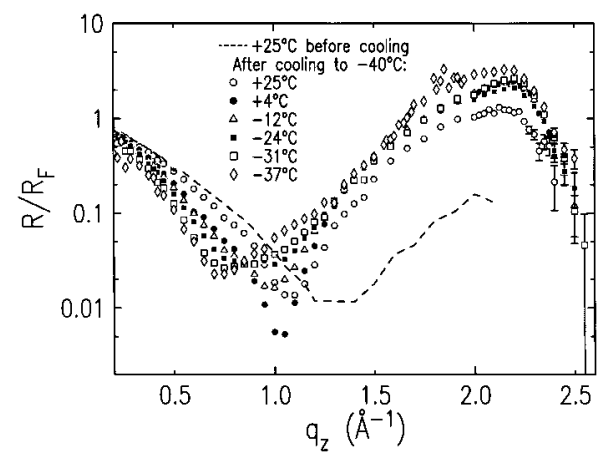

Figure 6. Fresnel-normalized reflectivity for $\mathrm{Hg} 0.20$ atom \% $\mathrm{Au}$ : $(---)+25^{\circ} \mathrm{C}$, before cooling the sample. Symbols: Data acquired after initally cooling to $-39{ }^{\circ} \mathrm{C}$, warming and maintaining at room temperature for $50 \mathrm{~h}$, and cooling a second time.

density profile having an additional low-density term positioned near the liquid-vapor interface, as described by eq 12 . Since this is the parametrization used previously in studies of pure liquid $\mathrm{Hg},{ }^{12}$ we can compare the pure and alloyed systems quantitatively. The layer spacing $d$, the thermal roughness $\sigma_{\mathrm{T}}$, and the decay parameter $\bar{\sigma}$ are parameters which characterize the layered subsurface structure and are all well determined by the fits. Other parameters affect the structure within about $2 \AA$ of the interface and are somewhat less reliably determined. The measured reflectivity curves that lack the prominent layering peak have been fit with a simpler model consisting of an error function profile along with an extra Gaussian term, as in eq 8 . Because of the low $\mathrm{Au}$ concentrations, the nearly equal scattering factors of $\mathrm{Hg}$ and $\mathrm{Au}$, and the limited $q_{z}$ range (over which the form factor varies slowly), we will treat our model profiles in this study as though they were entirely composed of $\mathrm{Hg}$. All models incorporate the bulk density and atomic form factor of $\mathrm{Hg}$ and therefore represent an average surface-normal electron density without providing any direct information about the Au composition as a function of depth.

B. Dilute Au Limit and Capillary Wave Roughness. At low $\mathrm{Au}$ concentrations, the main effect of $\mathrm{Au}$ alloying is to partially disrupt the layered profile near the surface, compared to the structure of $\mathrm{Hg}$. Model profiles for pure $\mathrm{Hg}$ at $+25^{\circ} \mathrm{C}$ (-) and $-36{ }^{\circ} \mathrm{C}(---)$, calculated from eq 12 in studies reported previously, ${ }^{12}$ are shown in Figure $7 \mathrm{a}$. The oscillatory profile has a layer spacing $d=2.72 \AA$, a decay parameter $\bar{\sigma}=$ $0.46 \AA$, and a temperature-dependent roughness $\sigma_{\mathrm{T}}$, given in Table 1, that determines the amplitude of the layers in the density profile. The model also incorporates a slight tail of density extending towards the vapor, described by the term proportional to $w_{\mathrm{A}}$ in eq 12 .

The surface-normal profile of $\mathrm{Hg} 0.06$ atom \% Au has the same layer spacing of $2.72 \AA$, but the layering is less well defined (Figire $7 \mathrm{~b}$ ). For comparable temperatures, the overall roughness $\sigma_{\mathrm{T}}$ is about $0.1 \AA$ greater than that of $\mathrm{Hg}$, producing a significant difference in layering amplitude. The layering also decays over a slightly shorter length scale for the alloy. Another notable difference between the structures of $\mathrm{Hg}$ and $\mathrm{Hg} 0.06$ atom $\% \mathrm{Au}$ is that for the alloy, the amplitude of the topmost surface layer is decreased by a factor of $w_{0}=0.8$ compared to pure $\mathrm{Hg}$, where $w_{0}=1$. The reduction of the second layer amplitude by $w_{1} \approx 0.95-0.98$ has a much smaller effect on the calculated reflectivity. These disruptions of the surface order are unsurprising in light of the different near-neighbor distances in bulk liquid $\mathrm{Hg}(3.0 \AA)$ and $\mathrm{Au}(2.8 \AA) .{ }^{34}$ As was found for $\mathrm{Hg},{ }^{12}$ the 0.06 atom \% alloy data are consistent with additional density in the near-surface region, described by the Gaussian term proportional to $w_{\mathrm{A}}$. 
TABLE 1: Parameters for Layered Density Profiles Having the Form Given in Eq 12, Where $\sigma_{n}^{2}=n \bar{\sigma}^{2}+\sigma_{\mathrm{T}}^{2}$ and $w_{n}=1$ Except As Indicated in the Table ${ }^{a}$

\begin{tabular}{|c|c|c|c|c|c|c|c|c|}
\hline atom $\% \mathrm{Au}$ & $T\left({ }^{\circ} \mathrm{C}\right)$ & $\sigma_{\mathrm{T}}(\AA)$ & $\bar{\sigma}(\AA)$ & $w_{1}$ & $w_{0}$ & $w_{\mathrm{A}}$ & $\sigma_{\mathrm{A}}(\AA)$ & $z_{\mathrm{A}}(\AA)$ \\
\hline 0 & +25 & $1.00 \pm 0.01$ & $0.46 \pm 0.05$ & 1.0 & 1.0 & $0.2 \pm 0.1$ & $1.5 \pm 0.5$ & $-2 \pm 1$ \\
\hline 0 & -3 & $0.87 \pm 0.01$ & $0.46 \pm 0.05$ & 1.0 & 1.0 & $0.2 \pm 0.1$ & $1.5 \pm 0.5$ & $-2 \pm 1$ \\
\hline 0 & -19 & $0.82 \pm 0.01$ & $0.46 \pm 0.05$ & 1.0 & 1.0 & $0.2 \pm 0.1$ & $1.5 \pm 0.5$ & $-2 \pm 1$ \\
\hline 0 & -36 & $0.80 \pm 0.01$ & $0.46 \pm 0.05$ & 1.0 & 1.0 & $0.2 \pm 0.1$ & $1.5 \pm 0.5$ & $-2 \pm 1$ \\
\hline 0.06 & +25 & $1.04 \pm 0.01$ & $0.54 \pm 0.04$ & $0.95 \pm 0.02$ & $0.80 \pm 0.02$ & $0.24 \pm 0.10$ & $1.6 \pm 0.5$ & $-2 \pm 1$ \\
\hline 0.06 & +11 & $1.02 \pm 0.01$ & $0.53 \pm 0.02$ & $0.96 \pm 0.02$ & $0.78 \pm 0.02$ & $0.24 \pm 0.10$ & $1.6 \pm 0.5$ & $-2 \pm 1$ \\
\hline 0.06 & -3 & $0.97 \pm 0.01$ & $0.50 \pm 0.02$ & $0.97 \pm 0.02$ & $0.78 \pm 0.02$ & $0.24 \pm 0.10$ & $1.6 \pm 0.5$ & $-2 \pm 1$ \\
\hline 0.06 & -26 & $0.91 \pm 0.01$ & $0.50 \pm 0.02$ & $0.98 \pm 0.02$ & $0.78 \pm 0.02$ & $0.24 \pm 0.10$ & $1.6 \pm 0.5$ & $-2 \pm 1$ \\
\hline 0.10 & +25 & $1.07 \pm 0.02$ & $0.52 \pm 0.02$ & $0.96 \pm 0.04$ & $0.84 \pm 0.04$ & $0.1 \pm 0.1$ & $2 \pm 1$ & $-2.6 \pm 0.5$ \\
\hline 0.10 & +5 & $0.99 \pm 0.01$ & $0.44 \pm 0.02$ & $0.96 \pm 0.04$ & $0.84 \pm 0.04$ & $0.4 \pm 0.2$ & $2 \pm 1$ & $-2.6 \pm 0.5$ \\
\hline 0.10 & -13 & $1.02 \pm 0.01$ & $0.43 \pm 0.02$ & $0.96 \pm 0.04$ & $0.78 \pm 0.03$ & $0.5 \pm 0.2$ & $2 \pm 1$ & $-2.6 \pm 0.5$ \\
\hline
\end{tabular}

${ }^{a}$ Data for pure $\mathrm{Hg}$, measured in the same UHV chamber, are from ref 12 . For all fits, $d=2.72 \pm 0.02 \AA$.

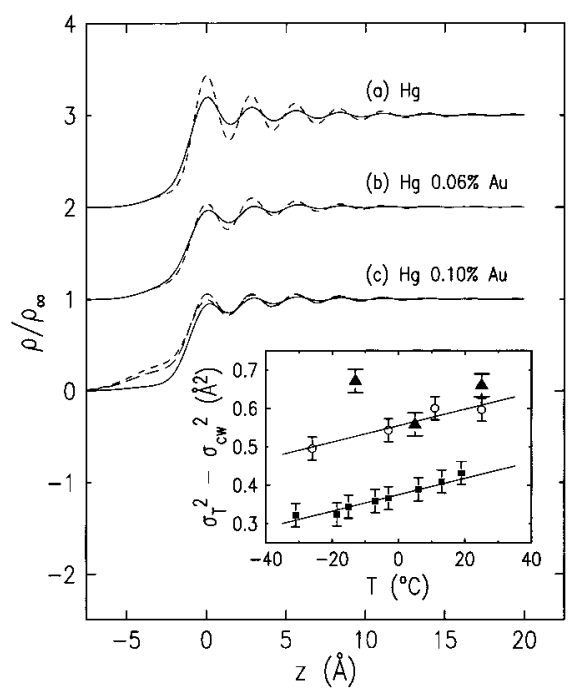

Figure 7. Model density profiles calculated from parameters listed in Table 1. (a) $\mathrm{Hg}$ : (-) $+25^{\circ} \mathrm{C}$; $(---)-36^{\circ} \mathrm{C}$. (b) $\mathrm{Hg} 0.06$ atom $\%$ $\mathrm{Au}:(-)+25{ }^{\circ} \mathrm{C} ;(---)-26{ }^{\circ} \mathrm{C}$. (c) $\mathrm{Hg} 0.10$ atom $\% \mathrm{Au}:(-)$ $+25{ }^{\circ} \mathrm{C} ;(---)+5{ }^{\circ} \mathrm{C} ;(--) \quad-13{ }^{\circ} \mathrm{C}$. Inset: temperature dependence of the excess mean-squared surface roughness $\left(\sigma_{\mathrm{T}}^{2}-\sigma_{\mathrm{cw}}{ }^{2}\right)$ for $\mathrm{Hg}(\square), \mathrm{Hg} 0.06$ atom \% $\mathrm{Au}(\mathrm{O})$, and $\mathrm{Hg} 0.10$ atom \% $\mathrm{Au}(\mathbf{\Delta})$.

The effect of temperature on the surface structure of $\mathrm{Hg}$ $0.06 \% \mathrm{Au}$ is very similar to the behavior found for pure $\mathrm{Hg}$. While slight variations in several parameters were required to optimize the fits at different $T$ for the $0.06 \%$ alloy, it is apparent that the main effect of increased temperature is to reduce the amplitude of the density oscillations. Capillary wave theory, which describes the broadening of the surface profile due to thermally excited surface waves, gives a specific prediction for the temperature behavior through the roughness $\sigma_{\mathrm{cw}}$, given in eq 3. Previous studies of the temperature-dependent surface roughness of liquid $\mathrm{Hg}$ found that thermally excited surface waves were not able to account for all of the temperature dependence observed. ${ }^{12}$ This is in contrast with the surface roughnesses of liquid $\mathrm{Ga}$ and $\mathrm{In}$, for which capillary wave theory accounted well for all the measured temperature dependence. ${ }^{14,15}$ In the inset of Figure 7 we compare the excess roughness $\sigma_{\mathrm{T}}{ }^{2}$ $-\sigma_{\mathrm{cw}}{ }^{2}$ of $\mathrm{Hg}$ with that of $\mathrm{Hg} 0.06$ atom \% Au. We see that the 0.06 atom \% alloy has a larger roughness, but the slope of the excess roughness versus $T$ is the same as for pure $\mathrm{Hg}$.

The fact that the deviation from capillary wave behavior is the same for pure $\mathrm{Hg}$ as for the 0.06 atom \% alloy, which has distinctly different intrinsic surface-normal structure, suggests that the deviation from capillary wave theory may not arise from a temperature dependence of the local profile. Instead, the apparently anomalous temperature dependence most likely arises from height fluctuations across the surface which are not fully accounted for by the capillary wave model. In our analysis, thermal capillary waves are dependent on a surface tension which is defined as a macroscopic quantity, despite the fact that we integrate over capillary wave modes extending down to atomic length scales. It is quite possible that the concept of a length-scale-independent surface tension is not applicable beyond some microscopic limit. If this is true then the success of capillary wave theory in the description of other liquids would stem from the fact that the modes at atomic length scales make up only a small part of the entire capillary wave spectrum. This hypothesis implies that the contribution of these modes in affecting the overall roughness may differ from system to system. Comparison of diffuse surface scattering from $\mathrm{Hg}$ and $\mathrm{Hg}-\mathrm{Au}$ alloys may provide more information on this issue.

The Au concentration of 0.10 atom $\%$ marks the transition between the dilute-Au regime, where a simple layered structure prevails, and the Au-rich phase. Here the more pronounced structure at low $q_{z}$ and the attenuated layering peak complicate the modeling, and the analysis is more ambiguous. For the given layered model, acceptable parameters lie in the ranges indicated in Table 1. Fit curves are shown in Figure 4c (solid lines). Relative to $\mathrm{Hg}$, the 0.10 atom \% alloy model density profiles exhibit a reduction in oscillation amplitude and surface layer density very similar to that found in the 0.06 atom $\%$ alloy, as shown in Figure 7c. Unlike the more dilute alloy, however, a more fundamental change in surface structure occurs as temperature is reduced beyond the solubility limit. Upon cooling from room temperature, the surface roughness first decreases, and then increases again, so that the layering peak is less pronounced at $-13{ }^{\circ} \mathrm{C}$ than it was at $+5{ }^{\circ} \mathrm{C}$. The resulting nonmonotonic dependence of $\sigma_{\mathrm{T}}$ on $T$ is shown in the inset of Figure 7 (closed triangles).

In addition, as $T$ decreases there is a progressive drop in the reflectivity at low $q_{z}$. This indicates a further decrease in the surface layer density, an enhanced density region further toward the vapor, or a combination of these effects. The density profiles shown in Figure 7c model this effect as a tail of density extending into the vapor region. This density tail is independent of temperature for $\mathrm{Hg}$ and $\mathrm{Hg} 0.06 \% \mathrm{Au}$, but for $\mathrm{Hg} 0.10 \% \mathrm{Au}$ it climbs steeply as the temperature is reduced. In this concentration and temperature range, the effect of decreased temperature is not a reduction in surface roughness but the establishment of a low-density surface region that destabilizes surface layering.

C. Au-Rich limit and Surface Phase Formation. The 0.13 atom \% Au alloy, near the room temperature saturation limit, can be modeled with a layered density profile only for our measurement at $+25^{\circ} \mathrm{C}$. Reflectivity data at lower temperatures exhibit a minimum at $q_{z} \sim 1.4-1.6 \AA^{-1}$, along with a slight dip at $q_{z} \sim 0.5 \AA^{-1}$. These features can be fit with the simple 


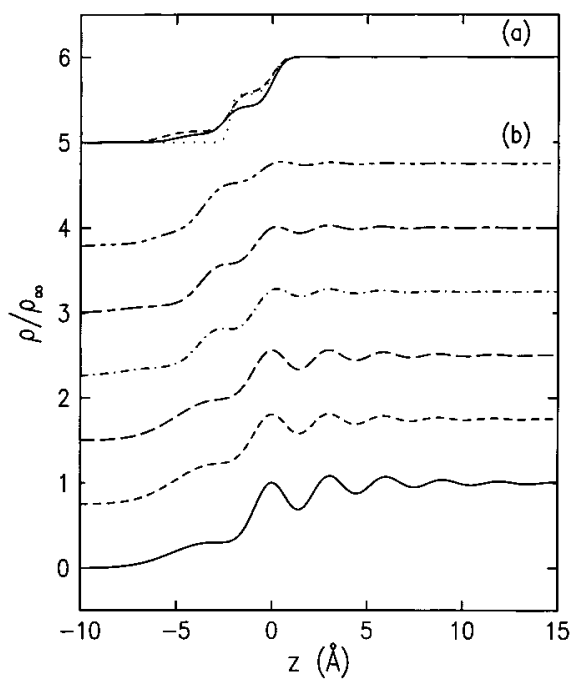

Figure 8. Model density profiles: (a) $\mathrm{Hg} 0.13$ atom $\% \mathrm{Au}:(-)+7$ ${ }^{\circ} \mathrm{C} ;(--)-18{ }^{\circ} \mathrm{C} ;(\cdots)-67{ }^{\circ} \mathrm{C}$. (b) $\mathrm{Hg} 0.20$ atom \% Au: (- --$)$ $+25{ }^{\circ} \mathrm{C} ;\left(--_{-}\right)+16{ }^{\circ} \mathrm{C} ;\left(-\cdot-_{-}\right)+8{ }^{\circ} \mathrm{C} ;(---)-20{ }^{\circ} \mathrm{C} ;\left(--_{-}\right)$ $-24{ }^{\circ} \mathrm{C} ;(-)-27^{\circ} \mathrm{C}$.

shelf-type models shown in Figure 8a. The relatively high reflectivity at the highest $q_{z}$ requires an unphysically low surface roughness, not encountered in other liquid metals and alloys. While this is possible in principle, a much more likely explanation of the reflectivity is that some surface layering prevails in the 0.13 atom $\%$ alloy, too weak to produce an easily identifiable layering peak but still substantial enough to produce constructive interference at high $q_{z}$ and augment the reflectivity which would otherwise be reduced by the surface roughness. For this reason, we think that the very simple shelf profiles are likely not the best description of the reflectivity, and models such as those presented below for the 0.20 atom $\%$ alloy may be more realistic. Despite this, attempts to fit the 0.13 atom $\%$ alloy with simple layered models were unsuccessful, with unphysically small layer spacings of $d \approx 2.1 \AA$ required to produce the reflectivity minimum at a position in $q_{z}$ matching the experimental data. ${ }^{35}$

The concentration of 0.20 atom $\% \mathrm{Au}$ in $\mathrm{Hg}$ exceeds the solubility limit for all temperatures investigated. Reflectivity data for this alloy exhibit low- $q_{z}$ minima at all $T$, along with a layering peak at the lowest $T$. We have fit these data to the simple layered profiles shown in Figure 8b. To keep the number of fit parameters as small as possible, we have used eq 12 and fit the same number of parameters as were used for the dilute alloys. At the lowest temperatures, the surface layer spacing of $2.9 \AA$ for $\mathrm{Hg} 0.20$ atom \% Au is unambiguously distinguished from the $2.72 \AA$ layer spacing obtained for pure $\mathrm{Hg}$ and the dilute $\mathrm{Hg}-\mathrm{Au}$ alloys. Since the near-neighbor distance in liquid $\mathrm{Au}(2.8 \AA)$ is smaller than that of $\mathrm{Hg}(3.0 \AA),{ }^{34}$ this increase in the layer spacing for the Au-rich alloy indicates that a significant change in packing has occurred in the surface layers. At higher temperatures, evidence for layering lies mainly in the relatively large reflectivity obtained for $q_{z} \geq 2 \AA^{-1}$. Here the fits shown in Figure 5c are less satisfactory, and the details of the models shown in Figure 8b exhibit no clear systematic dependence on temperature. While the oscillations in the density profile clearly grow as temperature is reduced, it is not possible to say to what extent this is due to reduction of surface fluctuations rather than to changes in Au content at the surface. Measurement techniques with elemental specificity, such as resonant X-ray scattering or surface X-ray absorption fine structure spectroscopy, will be required to obtain this chemical information. Because of the high vapor pressure of liquid $\mathrm{Hg}$, Auger electron spectroscopy will probably not be possible.

A feature common to all density profiles refined for the Aurich phase is the "shelf" feature or surface layer that has a density of about half that of the bulk; we were unable to model the notch in the reflectivity without such a feature. Presumably, some fraction of the Au which is insoluble in the bulk is incorporated into this surface phase, rather than precipitating out of the solution in solid form as might have been expected. It is difficult to explain how a monolayer composed entirely of $\mathrm{Hg}$ and/or Au might achieve this low density. All known $\mathrm{Hg}-$ Au phases are close-packed, with densities intermediate between those of elemental $\mathrm{Au}$ and $\mathrm{Hg} .{ }^{4} \mathrm{~A}$ uniform submonolayer of Au with a greatly expanded lateral spacing is one model which could produce the observed density profile. However, it is not clear how such an expanded phase, which would have a very high surface corrugation, could be stable given the range of temperature, pressure, and surface tension in our experiment. One possible contribution to an expanded spacing is that large changes in the concentration of $\mathrm{Au}$, which has a lower valence than $\mathrm{Hg}$, could reduce the bandfilling in the surface layers. If this serves to greatly reduce the favored atomic coordination, the surface atoms might be forced to adopt a more open structure. This picture is qualitatively consistent with the expansion of the surface-normal layer spacing in the Au-rich phase. Previous studies of liquid Ga and In surfaces showed that compared to the more covalent $\mathrm{Ga}$, the free-electron-like In had a more compressed surface layer spacing, indicating a structure closer to hard-sphere packing. ${ }^{15}$ However, it is hard to see how these effects, which are subtle for In compared to $\mathrm{Ga}$, could produce such a drastic reduction in the surface layer density.

Another possible explanation is that patches of more closely packed Au-rich monolayers float on the surface, comprising a coverage of about $50 \%$. We believe that detailed considerations argue against this model. Liquid $\mathrm{Hg}$ has been observed to amalgamate continuously with $\mathrm{Au}$ substrates, ${ }^{8}$ suggesting that it would be difficult to maintain a laterally inhomogeneous distribution of $\mathrm{Au}$ at the surface. Another objection is that if Au were precipitating out of solution and into a separate surface monolayer, we would expect a more monotonic change in coverage with temperature or concentration. Instead, the surface layer density seems to drop quickly to $50 \%$ of the bulk value and remain at that value. Finally, we find no evidence that the surface layer is solid. ${ }^{37}$

We now consider the possibility that some low-density impurity, such as oxygen or sulfur, is incorporated into the surface layer. It is important to emphasize that such impurities would also have been present in the pure $\mathrm{Hg}$ samples studied previously. While the pure $\mathrm{Hg}$ reflectivity provides evidence for a sample-dependent tail of density near the surface, as discussed above, nothing like the shelf feature, or layer having half the bulk density, was ever observed. In the case of the Aurich liquid alloys presented here, the shelf feature depends entirely on the presence of $\mathrm{Au}$ at the surface: only at temperatures and concentrations beyond the solubility limit does this structure appear. In contrast to pure $\mathrm{Hg}$, the Au-rich amalgam surface may be capable of forming a passivated oxide, even though elemental $\mathrm{Au}$ is quite nonreactive, and bulk $\mathrm{Hg}$ oxide is unstable under the low oxygen partial pressure. If this scenario is correct, this result may be relevant to reactions catalyzed by liquid metal surfaces. Studies of the rate of formic acid decomposition over liquid $\mathrm{Hg}$ having different concentrations of dissolved metals have found that the addition of higher- 
valence metals decreases the activation energy of the reaction. ${ }^{10}$ Our observations on the $\mathrm{Hg}-\mathrm{Au}$ alloys indicate that even in very dilute systems, the formation of intermetallic phases at the surface may be relevant. At the same time, the surface-induced layering intrinsic to all liquid metals may be less important for surface reactions, except for the extent to which the layering competes with the formation of the reactive surface phase.

\section{Conclusions}

We have used synchrotron X-ray reflectivity to determine the surface-normal structure of liquid $\mathrm{Hg}-\mathrm{Au}$ alloys near the room temperature solubility limit of 0.14 atom $\% \mathrm{Au}$ in $\mathrm{Hg}$. Alloys having $\mathrm{Au}$ concentrations below the solubility limit exhibit surface-induced layering similar to that found in pure $\mathrm{Hg}$ and other elemental liquid metals. Compared to pure $\mathrm{Hg}$, layering in the dilute $\mathrm{Hg}-\mathrm{Au}$ alloys is characterized by a significantly higher roughness, due to the difference in size of $\mathrm{Hg}$ and $\mathrm{Au}$ ions, which disrupts the layering. The temperature dependence of the surface structure in the dilute alloys deviates from the prediction of capillary wave roughening, exactly as was found for pure $\mathrm{Hg}$. At higher Au concentrations and lower temperatures, where the Au solubility limit is exceeded, we observe the formation of a new surface phase, with a significant change in packing of the ions at the surface.

To our knowledge, this work presents a potentially unique application of surface X-ray scattering to probe the bulk solubility behavior of a liquid alloy. This is of particular importance, since the most reliable methods for determining solubilities in dilute alloys are electroanalytical techniques. These techniques are not applicable to alloys such as $\mathrm{Au}$ in $\mathrm{Hg}$, where the solute is more noble than the solvent, ${ }^{20}$ unless they are modified by probing the formation of intermetallic compounds with a second added solute such as $\mathrm{Zn}$. For cases such as $\mathrm{Hg}-\mathrm{Au}$, where formation of a surface phase signals that the solubility limit has been exceeded, atomic-scale surface structural measurements should be of particular value. This study also shows that very dilute impurities in liquid metals can produce pronounced changes in the structure at the surface, where the composition can differ substantially from the bulk. Such structural and compositional changes may have pronounced effects on reactions taking place at liquid metals surfaces, including variations in catalytic and electrochemical systems which have been previously observed.

Acknowledgment. The authors acknowledge the contribution of O. M. Magnussen to the early stages of this study. This work is supported by U.S. DOE Grant Nos. DE-FG02-88ER45379 and DE-AC02-98CH10886, and by the U.S.-Israel Binational Science Foundation, Jerusalem. H.T. acknowledges support from the Deutsche Forschungsgemeinschaft.

\section{References and Notes}

(1) Aitchison, L. A History of Metals; Interscience: New York, 1960.

(2) Habashi, F. Principles of Extractive Metallurgy; Gordon and Breach: New York, 1969.
(3) Lappalainen, R.; Yli-Upro, A. Scand. J. Dent. Res. 1987, 95, 364. Oden, A.; Tulberg, M. Acta Odontol. Scand. 1985, 43, 15.

(4) Rolfe, C.; Hume-Rothery, W. J. Less-Common Met. 1967, 13, 1.

(5) Yang, X. M.; Tonami, K.; Tagahara, L. A.; Hashimoto, K.; Wei, Y.; Fujishima, A. Surf. Sci. 1994, 319, L17.

(6) Battistoni, C.; Bemporad, E.; Galdikas, A.; Kačiulis, S.; Mattogno, G.; Mickevičius, S.; Olevano, V. Appl. Surf. Sci. 1996, 103, 107.

(7) Nowakowski, R.; Kobiela, T.; Wolfram, Z.; Duś, R. Appl. Surf. Sci. 1997, 115, 217.

(8) Li, J.; Abruña, H. D. J. Phys. Chem. B 1997, 101, 2907.

(9) Kemula, W.; Kublik, Z.; Galus, Z. Nature 1959, 184, B.A.56.

(10) Schwab, G.-M. Ber. Bunsenges. 1976, 80, 746.

(11) Magnussen, O. M.; Ocko, B. M.; Regan, M. J.; Penanen, K.; Pershan, P. S.; Deutsch, M. Phys. Rev. Lett. 1995, 74, 4444.

(12) DiMasi, E.; Tostmann, H.; Ocko, B. M.; Pershan, P. S.; Deutsch, M. Phys. Rev. B 1998, 58, R13419.

(13) Regan, M. J.; Kawamoto, E. H.; Lee, S.; Pershan, P. S.; Maskil, N.; Deutsch, M.; Magnussen, O. M.; Ocko, B. M.; Berman, L. E. Phys. Rev. Lett. 1995, 75, 2498.

(14) Regan, M. J.; Pershan, P. S.; Magnussen, O. M.; Ocko, B. M.; Deutsch, M.; Berman, L. E. Phys. Rev. B 1996, 54, 9730.

(15) Tostmann, H.; DiMasi, E.; Pershan, P. S.; Ocko, B. M.; Shpyrko, O. G.; Deutsch, M. Phys. Rev. B 1999, 59, 783.

(16) Rice, S. A. J. Non-Cryst. Solids 1996, 205-207, 755, and references therein.

(17) Regan, M. J.; Pershan, P. S.; Magnussen, O. M.; Ocko, B. M.; Deutsch, M.; Berman, L. E. Phys. Rev. B 1997, 55, 15874.

(18) Lei, N.; Huang, Z.; Rice, S. A. J. Chem. Phys. 1997, 107, 4051.

(19) Tostmann, H.; DiMasi, E.; Shpyrko, O. G.; Pershan, P. S.; Ocko,

B. M.; Deutsch, M. Ber. Bunsenges. Phys. Chem. 1998, 102, 1136.

(20) Gumiński, C. J. Less-Common Met. 1991, 168, 329.

(21) Braslau, A.; Pershan, P. S.; Swislow, G.; Ocko, B. M.; Als-Nielsen,

J. Phys. Rev. A 1988, 38, 2457.

(22) Deutsch, M.; Ocko, B. M. Encycl. Appl. Phys. 1998, 23, 479.

(23) Au: Johnson Matthey, 20 mesh, 99.9995\%; Hg: Bethlehem Apparatus Co., quadruple distilled, 99.9999\%.

(24) Encyclopedia of Electrochemistry of the Elements; Bard, A. J., Ed.; Marcel Dekker: New York, 1973; Vol. IX, Part A, p 31.

(25) Thomas, B. N.; Barton, S. W.; Novak, F.; Rice, S. A. J. Chem. Phys. 1987, 86, 1036.

(26) Lu, B. C.; Rice, S. A. J. Chem. Phys. 1978, 68, 5558.

(27) Als-Nielsen, J. In Structure and Dynamics of Surfaces II; Schomers, W.; von Blanckenhagen, P., Eds.; Springer: Heidelberg, Germany, 1987. (28) Chason, E.; Mayer, T. M. Crit. Rev. Solid State Mater. Sci. 1997, 22,1 .

(29) Bunker, B. A.; Kropf, A. J.; Kemner, K. M.; Mayanovic, R. A.; Lu, Q. Nucl. Instrum. Methods Phys. Res. B 1997, 133, 102.

(30) Pershan, P. S. Phys. Rev. E 1994, 50, 2369.

(31) The expression in eq 3 is given incorrectly in eq 12 of ref 15 .

(32) Warren, B. E. X-ray Diffraction; Addison-Wesley: Reading MA, 1969

(33) Kertes, A. S., Ed. Metals in Mercury; Solubility Data Series; Pergamon Press: New York, 1986; Vol. 25, p. 378.

(34) Iida, T.; Guthrie, R. I. L. The Physical Properties of Liquid Metals; Clarendon Press, Oxford, UK, 1993; p 35.

(35) The surface roughness in the step-type profile was especially low for the $\mathrm{Hg} 0.13$ atom $\%$ Au sample supercooled to $-67{ }^{\circ} \mathrm{C}$, the lowest temperature obtainable in our chamber. The 0.13 atom \% Au alloy is the only one for which we observed deep supercooling: all other alloys investigated froze at temperatures close to the melting point of pure $\mathrm{Hg}$, $-38.9^{\circ} \mathrm{C}$. We cannot tell whether the supercooling is particular to the 0.13 atom \% Au composition or is due instead to an especially clean sample environment. It has been suggested before that supercooling at an ordered liquid metal surface may be enhanced due to the reduction of the difference in entropy between the liquid and solid phases relative to a disordered surface (ref 36). However, without more systematic studies of supercooled samples it does not seem worthwhile to try to interpret this observation.

(36) Turnbull, D. J. Chem. Phys. 1952, 20, 411.

(37) Grazing incidence diffraction measurements, which probe in-plane correlations of the first $50 \AA$ at the surface, were performed for the $\mathrm{Hg}$ 0.13 atom $\%$ Au alloy at room temperature and at $-20{ }^{\circ} \mathrm{C}$. No sharp peaks indicative of in-plane order at the surface were observed; the data contained only features characteristic of the bulk $\mathrm{Hg}$ structure factor. 